\title{
Prospective Observation of Switching Rate of Antidepressants in Management of Depressive Episode in 3 Months
}

\author{
Weidong Jin", Naixin Wang, Yongchun Ma, Zhenhua Tong \\ Tongde Hospital of Zhejiang Province, Hangzhou, China \\ Email: "wdjin@163.com
}

Received June $25^{\text {th }}, 2012$; revised January $5^{\text {th }}, 2013$; accepted February $7^{\text {th }}, 2013$

\begin{abstract}
Objective: to observe prospectively of switching rate of antidepressants in management of depressive episode. Methods: 190 patients with depressive episode treated by antidepressants were observed for 3 months and switching rate were assessed under "best natural therapy". And switching criteria was following: 1) manic or hypomanic episode; or 2) promoting rapid cycling; or 3) YMRS $\geq 11$ or irritability or aggravation in YMRS $\geq 3$. 4) Psychiatrist thinks that the patients should be stopped to take antidepressants and should be placed by mood stabilizer or antipsychotics or their combination. Results: 1) 18 of 190 patients was found to switch in 3 month therapy. The switching rate was $9.47 \%$. 2) 4 of 61 males was found and 14 females was found to switch, their switching rate was not significant $\left(6.56 \%, 10.85 \%, \mathrm{X}^{2}=0.89, P\right.$ $>0.05$ ). 3) 10 of 170 unipolar depression and 8 of bipolar depression was found to switch, their switching rate was very significant $\left(5.88 \%, 40 \%, X^{2}=24.29, P<0.01\right)$. 4) 5 of 36 patients token mood stabilizer and 13 patients not token mood stabilizer was found to switch, their switching rate was not significant $\left.\left(13.8 \%, 8.44 \%, X^{2}=1.47, P>0.05\right) .5\right) 7$ of 54 patients token more two antidepressants and 11 patients token single antidepressant was found to switch, their switching rate was not significant $(12.96 \%, 8.08 \%$, $\left.\mathrm{X}^{2}=1.07, P>0.05\right)$. 6) 7 of 38 patients with family history of mood disorder and 11 of patients without family history of mood disorder was found to switch, their switching rate was significant $(18.4 \%, 7.23 \%$, $\left.\mathrm{X}^{2}=4.43, P<0.05\right) .7$ ) The switching often carried out at one month after treatment with antidepressants. 8) The significant difference in switching rate among various antidepressants were not found. Conclusion: Some depressive patients may switch during treatment with antidepressant, it should be stressed on.
\end{abstract}

Keywords: Antidepressants; Switching; Bipolar Depression; Family History; Mood Stabilize

The concept of bipolar disorder has expanded from one in which there are major pathological variations in mood of a severe, extreme form ,to one in which more subtle variation are found, which merger imperceptibly with "normal" mood variation. These milder forms appear to be common. They may become more severe under some circumstances and show some degree of progression. The triggers for such progression can be socially, or hormonally, or pharmacologically, especially antidepressants mediated (Ferrier, Macmillan, \& Yong, 2011). These patients can belong to bipolar spectrum disorder, which is bigger diagnostic fields consisting of many disorder (Akiskal, 1996). And it also can be thought as soft bipolar (Jin, Chen, \& Xing, 2005; Jin, Chen, \& Xing, 2007a), which have been studied and discussed by Akiskal and others. The relation of these clues to bipolarity also documented in some Chinese literatures (Jin et al., 2005, 2007). Several studies have advocated including patients with antidepressant-induced mania or hypomania in the bipolar spectrum (Ghaemi, Ko, \& Goodwin, 2002). Akiskal has also note that, when followed prospectively, many adults patients with antidepressant-associated hypomania are found to progress to bipolar states with spontaneous mania or hypomania months or years later. In fact, treatment-induced hypomania was $100 \%$ specific for the eventual endpoint of bipolar disorder, closely followed by a family history of bipolar disorder, which

\footnotetext{
*Supported by Medicine study Medicine fund of Zhejiang Province (No 2006A017), China.

${ }^{\#}$ Corresponding author.
}

was $98 \%$ specific. Consequently, we give greater weight to these 2 factors as predictive of a bipolar illness (Ghaemi, Ko, \& Goodwin, 2002).

The nature of depressive episode determines the diversity of effects of antidepressant treatment variability, particularly significant reaction differences often appear between unipolar depression and bipolar depression Therapy differences were apparent, so many depression treatment guidelines emphasize the distinction .Because some patients with depression present exciting performance in the role of antidepressants, the socalled "switching", in which case there's change we call turn to manic state, such as antidepressants cause mania or hypomania, also so called BP-III patients, Bipolar III disorder (pseudounipolar bipolar disorder) refer to a more heterogeneous grouping of people who experience recurrent episodes of unipolar depression and also show clinical features suggesting that they may go on to development a hypomanic or manic episode. Such people may have a family history of bipolar disorder, experienced antidepressant-induced hypomanic switching, or have hyperthymic, dysthymic or cyclothymic, premorbid temperaments (Ferrier, Macmillan, \& Yong, 2011; Akiskal, 1996; Jin, Chen, \& Xing, 2007b). However, it has been controversial that how much prevalence of antidepressants triggering switch during treatment. Because this switching involved the unmber of chance in the end, such as definition of switching (Jin, Chen, \& Xing, 2007a), time of administration (Jin, Chen, \& Xing, 2007b), drug type (Jin X. G. \& Jin W. D., 2007), type of dis- 
ease and to investigate or research methods (Jin X. G. \& Jin W. D., 2007).Most of current studies is a retrospective investigation (Akiskal, 1996; Jin, Chen, \& Xing, 2007a; Jin X. G. \& Jin W. D., 2007), and prospective studies is less, which can be right investigating un-controlling factors of duration of therapy, combination therapy problems in the nature of the care status. Prospective study that we apply may be effective in controlling certain variables, in order to more accurately at same time observed certain drugs trigger or induce switching and it's incidence. We conducted research in this area, and will report is as following.

As episode of depression, especially first episode depression, was difficult to distinguished as unipolar or bipolar, and because $2 / 3$ bipolar disorder always had depression episode as first onset, and most depression was common clinical phase during all of bipolar disorder, and also unipolar and bipolar depression was similar in psychopathology. So whereas unipolar or bipolar depression, only depression episode, was companied mania, which was induced by antidepressants or naturally appearance, as has significant clinical role. This means that patients should be diagnosed as bipolar disorder. So putting stress on switching is very important. The same case is caring switching rate in clinical. During median follow-up of 41 weeks (range, 8 - 251 weeks), manic conversion occurred in 4786 patients $(5.4 \%)$. Multivariate analyses using time-dependent Cox proportional hazards models indicated that an increased risk of manic conversion was associated with antidepressant category vs no antidepressant exposure (hazard ratios: 2.1 for selective serotonin reuptake inhibitors, $P<.001$; 3.8 for "other" antidepressants, $P<.001$; and 3.9 for tricyclic antidepressants, $P=.002$ )

\section{Material and Methods}

Study of 190 cases met the American standards of classification and diagnosis of mental disorders fourth edition (DSM-IV) and the Chinese classification and diagnostic criteria for mental disorders third edition (CCMD-3) in the depressive episode or bipolar depression diagnostic criteria. Male patients was 61 cases, female was 129 cases, aged was from 16 to 62 years old, age mean was $25.6 \pm 19.9$ years. There were 170 cases of depression, bipolar depression, 20 cases in 190 patients; a positive family history of mood disorder were 38 cases and 152 patients without family history of mood disorders.

Switching criteria: 1) to appear manic or hypomanic episode; or 2) to become quicker circulation of the originals; or 3) Young Mania Rating Scale $\geq 11$ points or one of irritability and destruction attacks $\geq 3$ points; or 4 ) therapists believe that the use of antidepressants should be discontinued antipsychotics or mood stabilizers or change their therapeutic methods.

Treatment: Each patient diagnosed with depression or bipolar depression was managed by psychiatrists with the most natural treatment. The most natural is the so-called that doctors use of antidepressant drugs for the patient based on the diagnosis and their own treatment experience of adding or not adding mood stabilizers. However, the patient wasn't observed that added typical or atypical antipsychotics. Duration observed continue for 3 months. In 3 months was continuous treatment, without interruption and not to change antidepressants.

Evaluation: Three scales were sued. There were Hamilton Depression Rating Scale (HAMD), Hamilton Anxiety Rating
Scale (HAMA) Young Mania Rating Scale (YMRS). Patients were assessed before treatment and after 1 week, 1 month, 2 months, in March. No matter how the ratings, 3 require a scale of assessment. When a patient at some time within 3 months is considered the switching, the subsequent assessment of the interruption and as a "positive" patients treated.

\section{Statistics}

Application of Chi-square test in SPSS10.0 for $\mathrm{X}^{2}$ test.

\section{Result}

1) Switching rate: There were 18 patients switching to mania during therapy for 3 months. The switching rate was $9.47 \%$.

2) Switching time: The time to switching during treatment was from shortest 3 days to longest 81 days, and average was $28.4 \pm 24.5$ day. And there was no difference between male and female patients $(30.8 \pm 19.5$ vs $26.9 \pm 13.1$ day $)$, but it's was longer of unipolar patients than that of bipolar patients $(45.9 \pm$ 8.1 vs $20.4 \pm 10.6$ day), and it's shorter of patients with family history of bipolar disorder than that without family history of bipolar disorder $(23.5 \pm 12.8$ vs $33.7 \pm 20.1$ day $)$.

3) Comparison relatively as following.

4) Comparison between different antidepressants.

There were 136 patients treated by only one antidepressant and 11 cases switched. The number of 80 patients treated with SSRI switched was $5(6.25 \%)$, of 26 patients with SNRI switched was $3(11.5 \%)$, of 25 patients with TCA switched was $2(8 \%)$, of 5 patients with others switched was $1(20 \%)$. No differences in switching rate was found $\left(\mathrm{X}^{2}=2.21, P>.05\right)$. If TCA was thought as dual action mechanism antidepressant as SNRI, no difference in switching rate between SSRI and dual action mechanism antidepressants (TCA + SNRI, $\left.\mathrm{X}^{2}=0.55, P>.05\right)$.

Table 1.

Comparison relative factors.

\begin{tabular}{|c|c|c|c|c|c|}
\hline Factors & Cases & $\begin{array}{l}\text { Cases of } \\
\text { switching }\end{array}$ & $\begin{array}{c}\text { Switching } \\
\text { rate }\end{array}$ & $X^{2}$ & $P$ \\
\hline \multicolumn{6}{|l|}{ Gender } \\
\hline Male & 61 & 4 & $6.56 \%$ & 0.89 & $>.05$ \\
\hline Female & 129 & 14 & $10.85 \%$ & & \\
\hline \multicolumn{6}{|l|}{ Phase } \\
\hline Unipolar & 170 & 10 & $5.88 \%$ & 24.29 & $<.01$ \\
\hline Bipolar & 20 & 8 & $40.00 \%$ & & \\
\hline Family & History & of & Mood & Disorders & \\
\hline Positive & 38 & 7 & $18.40 \%$ & 4.43 & $<.05$ \\
\hline Negative & 152 & 11 & $7.23 \%$ & & \\
\hline \multicolumn{6}{|c|}{ Antidepressants } \\
\hline Only & One & 136 & 11 & $8.08 \%$ & 1.07 \\
\hline Two & And & More & 54 & 7 & $12.96 \%$ \\
\hline Mood & Stabilizer & & & & \\
\hline Yes & 36 & 5 & $13.80 \%$ & 1.47 & $>.05$ \\
\hline No & 154 & 13 & $8.44 \%$ & & \\
\hline
\end{tabular}




\section{Discuss}

On the antidepressant drug-induced phase conversion of most of the data are retrospective, and therefore to some extent there may be partial differences. Therefore, prospective observation may avoidance this partial differences, which can find real switching rate under certain the conditions specified or natural treatment condition in a certain time period of antidepressant therapy and can find risks related to switching. Our results show that about $10 \%$ depressive episode patients may switch and it often appear at about 1 month after beginning of treatment.

Clinicians managing depression in patients with bipolar disorder or patients who we don't know its trait face two challenges. First, major depression is a handicapping disease and is associated with a high risk of suicide in bipolar patients. Second, in bipolar patients, antidepressant drugs are associated with a high risk of antidepressant-induced mania (AIM) and rapid cycling. The definition of predictors of AIM is a key issue for the therapeutic management of bipolar patients. Clinical studies on bipolar patients with and without AIM have identified characteristics with poor sensitivity and low specificity. Many factors related to switching was found, such as family history of bipolar disorder, early onset of disorder, hyperthemia of Temperament, extreme personality, history of switching, and some biological factors, such as Hypothyroidism, functional polymorphism of the serotonin transporter (5-HTT) gene (Rousseva et al., 2003). Also findings suggesting gender, some clinical features (Jin et al., 2006), dysthmia and cyclothmia, or borderline personality disorder (Ghaemi, Ko, \& Goodwin, 2002 ,) maybe related to switching. But all these refer to bipolar disorder. In fact, some depressive patients diagnosed as unipolar depression maybe switched during treatment with antidepressants.

Tondo L. (2009) review available data pertaining to risk of mania-hypomania among bipolar (BPD) and major depressive disorder (MDD) patients with vs. without exposure to antidepressant drugs (ADs) and consider effects of mood stabilizers, They computerized searching yielded 73 reports (109 trials, 114,521 adult patients); 35 were suitable for random effects meta-analysis, and multivariate-regression modeling included all available trials to test for effects of trial design, AD type, and mood-stabilizer use. And found that overall risk of mania with/without ADs averaged 12.5\%/7.5\%. The AD-associated mania was more frequent in BPD than MDD patients (Tondo, Vázquez, \& Baldessarini, 2010). We also had some results. 10 of 170 unipolar depression had switched during antidepressants therapy. It indicated that there is greater difference in switching rate between bipolar and unipolar disorder, which was most significant risk for switching. These patients can not be diagnosed as bipolar disorder by model criteria. when followed prospectively, many adults patients with antidepressant-associated hypomania are found to progress to bipolar states with spontaneous mania or hypomania months or years later (Ghaemi, Ko, \& Goodwin, 2002; Gao et al., 2008). So the patients with switching of mania could be diagnosed bipolar disorder only depending on modification of model bipolar disorder diagnose criteria (Jin et al., 2006; Chun \& Dunner, 2004; Gao et al., 2008).

In General, Combination usage of antidepressant may one risk for switching (Jin, Chen, \& Xing, 2007a; Gao et al., 2008), but we don't found this conclusion, which may be related to short duration of observation and less cases. Many results supported that 2 or more AD combination for treatment of depressive episode promoted risk of switching to mania or rapid cycling. But this switching can be interrupted or reduced by mood stabilizers, it's risk can be reduced $50 \%$ (Bottlender et al., 2001). In past we also found lithium carbonate can significantly reduced switching risk in bipolar disorder (Jin X. G. \& Jin W. D., 2007). But our result can not show the same result, which may be related more "nulpolar" depression patients who be treated less mood stabilizer and all patient be treated less mood stabilizer. Only 5 patients in our studied patients were congratulated with mood stabilizes, which reflected therapy status of psychiatrist in china.

Treatment of bipolar depression patients is caused for concern, In general, antidepressants do not need the participation of many bipolar depression treatment Many guidelines also emphasize this point. But most of the cases, the risk of bipolar depression phase conversion was significantly higher than unipolar depression, almost close to half of depression may converse to drug-related mania or hypomania, even rapid cycling. Our previous study found that bipolar depression is occurred during treatment with probability close to $40 \%$ (Song et al., 2008), this study also suggest a similar discovery.

Different antidepressants maybe had a various switching rate for patients with depressive episode. In general, TCA and SNRI had more promotion to mania than that of SSRIs for depressive patients. So bipolar depressive patients shouldn't be treated with antidepressants except special conditions, in which only SSRIs was used. Some studies found that TCA and SNRI is more than motivational than SSRI, especially for bipolar depression on the types of antidepressant drugs (Peet, 1994; Barak, Kimhi, \& Weizman, 2000). The switching rate of TCA was 12 times compare to citalopram (Barak, Kimhi, \& Weizman, 2000), SNRI's switching rate of SSRI 3 times compare to paroxetine (Peet, 1996), we do not have similar findings, which may be and fewer cases and the observation time is short. But more of concern is the type of depression.

\section{REFERENCES}

Akiskal, H. S. (1996). The prevalent clinical spectrum of bipolar disorders: Beyond DSM-IV. Journal of Clinical Psychopharmacology, 16, $4 \mathrm{~s}-14 \mathrm{~s}$.

Barak, Y., Kimhi, R., \& Weizman, R. (2000). Is selectivity for serotonin uptake associated with a reduced emergence of manic episodes in depressed patients? International Clinical Psychopharmacology, 15, 53-56. doi:10.1097/00004850-200015010-00009

Bottlender, R., Rudolf, D., Strauss, A. et al. (2001). Mood stabilizers reduce the risk of developing antidepressant-induced maniform state in acute treatment of bipolar I depressed patients. Journal of Affective Disorders, 63, 79-83. doi:10.1016/S0165-0327(00)00172-5

Chun, B. J., \& Dunner, D. L. (2004). A review of antidepressant-induced hypomania in major depression: Suggestions for DSM-V. Bipolar Disorders, 6, 32-42. doi:10.1046/j.1399-5618.2003.00084.x

Ferrier, I. N., Macmillan, I. C., \& Yong, A. H. (2011). The search for the wadering thymostat: A rewiew of some developments in bipolar research. The British Journal of Psychiatry, 178, s103-s106.

Gao, K., Kemp, D. E., Ganocy, S. J. et al. (2008). Treatment-emergent mania/hypomania during antidepressant monotherapy in patients with rapid cycling bipolar disorder. Bipolar Disorders, 10, 907-915. doi:10.1111/j.1399-5618.2008.00637.x

Ghaemi, S. N., Ko, J. Y., \& Goodwin, F. K. (2002). Cade's disease and beyond: Misdiagnosis, antidepressant use, and a proposed definition for bipolar spectrum disorder. Canadian Journal of Psychiatry, 47, 
125-134.

Jin, W. D. (2007). Antidepressants and switching (in Chinese). Journal of Clinical Psychology in Medical Settings, 17, 209-221.

Jin, W. D., Chen, J., Xing, B. P., et al. (2007a). Antidepressants and switching (in Chinese). Joural of Clinical Psychology in Medical Setting, 17, 209-221.

Jin, W. D., Chen, J., Xing B. P. et al. (2005). Clinical epidemiological survey of switching induced by antidepressants. Switching rate and it's difference in different type of depression (in Chinese). Journal of Pharmacology Epidenmiology, 14, 217-219.

Jin, W. D., Chen, J., Xing, B. P. et al. (2007b). Establishment and evaluation of advising criteria of soft bipolar disorder (in Chinese). Chinese Journal of Behavioral Medical Science, 16, 801-803.

Jin, W. D., Tang, X. X., Wang, H. Q. et al. (2006). Meta-analysis of the difference in clinical symptomatology between Bipolar and Unipolar depression (in Chinese). Chinese General Practice, 9, 401-403.

Jin, X. G., \& Jin, W. D. (2007). Lithium carbonate can decrease switch- ing associated with antidepressants: Meta-analysis of domestic data. Shanghai Arch Psychiatry, 19, 313-314.

Peet, M. (1994). Induction of mania with selective serotonin re-uptake inhibitors and tricyclic antidepressants. The British Journal of Psychiatry, 164, 549-550. doi:10.1192/bjp.164.4.549

Rousseva, A., Henry, C., van den Bulke, D. et al. (2003). Antidepressant-induced mania, rapid cycling and the serotonin transporter gene polymorphism. The Pharmacogenomics Journal, 3, 101-104. doi:10.1038/sj.tpj.6500156

Song, Z. X., Xu, L. P., Jin, W. D. et al. (2008). Retrospective study on switching rate of different antidepressants in the treatment of bipolar depression (in Chinese). Journal of Psychiatry, 21, 9-13.

Tondo, L., Vázquez, G., \& Baldessarini, R. J. (2010). Mania associated with antidepressant treatment: Comprehensive meta-analytic review. Acta Psychiatrica Scandinavica, 121, 404-414. doi:10.1111/j.1600-0447.2009.01514.x 